\title{
Bmal1 deletion in mice facilitates adaptation to disrupted light/dark conditions
}

\author{
Guangrui Yang, ${ }^{1,2}$ Lihong Chen, ${ }^{3}$ Jiayang Zhang, ${ }^{3}$ Baoyin Ren, ${ }^{1}$ and Garret A. FitzGerald ${ }^{2}$ \\ 'School of Bioengineering, Dalian University of Technology, Dalian, Liaoning, China. ${ }^{2}$ Institute for Translational Medicine \\ and Therapeutics, Perelman School of Medicine, University of Pennsylvania, Philadelphia, Pennsylvania, USA. \\ ${ }^{3}$ Advanced Institute for Medical Sciences, Dalian Medical University, Dalian, Liaoning, China.
}

\begin{abstract}
Recently, by using conventional and tamoxifen-inducible brain and muscle Arnt-like protein 1knockout (Bmal1-KO) mice, we found that delaying the loss of circadian rhythms to adulthood attenuates the impact on general integrity and survival at least under 12-hour light/12-hour dark conditions. To understand further the contribution of Bmal1 in postnatal life under conditions of circadian disruption, we subjected inducible-KO and their littermate controls (ctrls) to forced desynchrony protocols, including cycles with non-24-hour periods, randomized light/dark cycles, and jet lag, and monitored their locomotor activity using radiotelemetry. Under these conditions, ctrl mice cannot be entrained, as reflected by their maintenance of circadian behavior irrespective of schedules. By contrast, $\mathrm{KO}$ mice displayed higher activity levels in the dark phases of most cycles. Under a 3-hour light/3-hour dark regime, ctrls displayed higher activity levels in the dark phases of all cycles, although there were still obvious circadian rhythms, suggesting that an ultradian mechanism is also involved. Insulin sensitivity was markedly reduced by disrupted light schedules, as expected in ctrls, but not in the KO mice. Thus, Bmal1 deletion in adult mice facilitates adaptation to new light/dark schedules and protects from insulin resistance induced by circadian disruption.
\end{abstract}

Authorship note: $\mathrm{GY}$ and $\mathrm{LC}$ contributed equally to this work.

Conflict of interest: GAF is a senior advisor to Calico. His laboratory receives funding support from the Volkswagen Foundation, the American Heart Association, and the NIH.

Copyright: (c) 2019 American Society for Clinical Investigation

Submitted: September 25, 2018

Accepted: April 4, 2019

Published: April 11, 2019.

Reference information: /CI Insight. 2019;4(10):e125133. https://doi. org/10.1172/jici.insight.125133.

\section{Introduction}

Chronic disruption of circadian rhythms may result in adverse effects in humans. For example, shift work has been associated with an increased burden of cardiometabolic disease, cancer, and sleep disorders (1). Although other factors, such as socioeconomic status and behavioral covariates, may confound such associations, circadian disruption in mice or in humans using forced desynchrony protocols results in analogous adverse phenotypes. Deletion of brain and muscle Arnt-like protein 1 (Bmal1), the only core clock gene whose loss results in complete absence of circadian rhythms, causes behavioral arhythmicity concomitant with many adverse effects, including reduced body weight, impaired hair growth, abnormal bone calcification, eye pathologies, and shortened life span (2-4). Selective deletion of Bmal1 in pancreas (5), liver (6), muscle (7), and adipose tissue (8) recapitulates discrete elements of the metabolic syndrome. This phenomenon is of increasing concern as circadian disruption becomes commonplace in modern life because of nocturnal use of illuminated phones, transmeridian flights, and shift work.

Recently, using tamoxifen-inducible (TAM-inducible) Bmall-knockout (Bmall-KO) mice, we found that although the mice lost behavioral, physiological, and molecular circadian rhythms, there was an attenuated impact on general integrity and survival under regular light/dark conditions (9). However, the effect of Bmall deletion in mice under disrupted light/dark schedules (DSs) has not been evaluated. Thus, we have subjected inducible Bmall-KO and their littermate controls to a series of non-24-hour light/dark cycles, to determine whether the absence of a molecular clock in adulthood might facilitate adaptation to circadian disruption.

\section{Results}

Loss of circadian behavior. Locomotor activity under the series of disrupted light/dark conditions (Figure 1) was monitored using radiotelemetry or running wheels. Before TAM treatment, there was no behavioral difference between Bmallfff Esr-Cre mice and their littermate Bmal1 ${ }^{f / f}$ controls under either 12-hour light/12-hour dark (LD) conditions or constant darkness (DD) conditions (Figure 2A). 


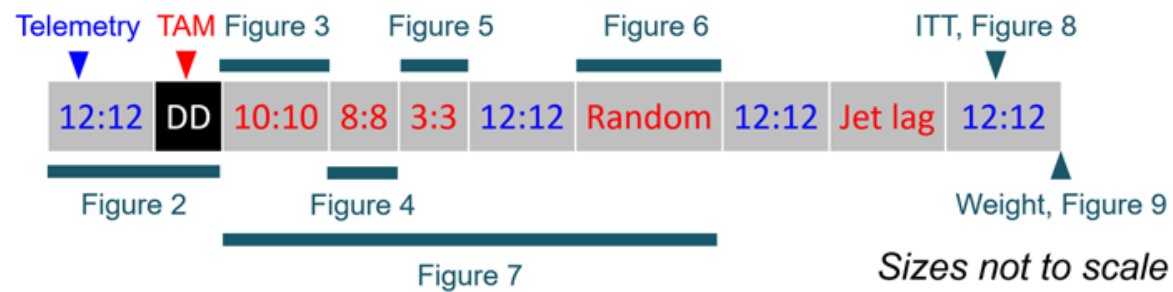

Figure 1. Study design. Telemetry-implanted mice were first maintained under 12-hour light/12-hour dark (LD), and then released to constant darkness (DD), during which they were treated with TAM. Two weeks later, mice were subjected to a series of disrupted light/dark schedules, including 10-hour light/10-hour dark, 8-hour light/8-hour dark, 3-hour light/3-hour dark, LD, randomized light/dark, LD, jet lag, and LD. Insulin tolerance test, body weight, and epididymal fat weight were measured after the series of schedules.

Bmalffff mice treated with TAM (Figure 2, A and B) and Bmal1 ${ }^{f / f}$ Esr-Cre mice treated with corn oil (Figure 2B) still showed rhythmic behavior under DD; however, as expected, all Bmallfff Esr-Cre mice treated with TAM completely lost the rhythmicity after the treatment. It is noteworthy that TAM, but not corn oil, decreased locomotor activity (Figure 2B). Therefore, we chose TAM-treated Cre mice as controls (ctrls) for the following experiments to avoid possible confounding effects caused by the different ingredients of the gavage.

Adaptation to various light/dark schedules. After DD, the mice were subjected to a series of abnormal light/dark schedules, including L/D $=10$ hours/10 hours (Figure 3), L/D = 8 hours/ 8 hours, (Figure 4), $\mathrm{L} / \mathrm{D}=3$ hours $/ 3$ hours (Figure 5 ), and a randomized light/dark condition (Figure 6 ). Save for the $\mathrm{L} / \mathrm{D}=$

A

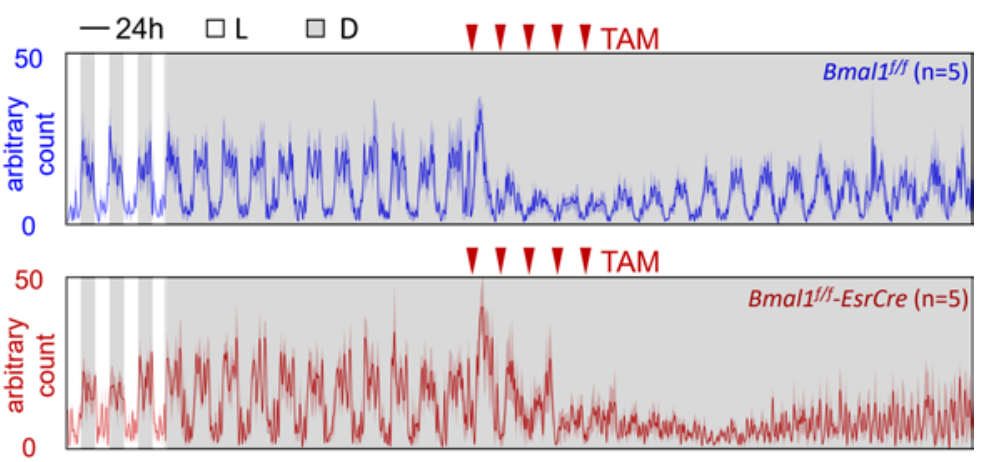

B
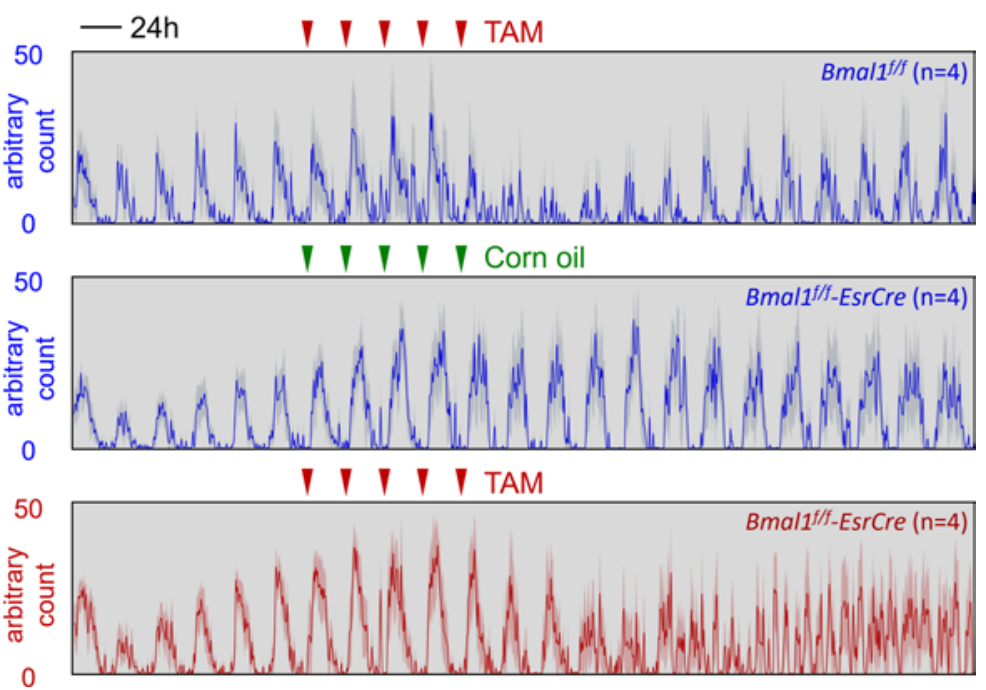

Figure 2. Locomotor activity under DD. (A) Telemetry-implanted mice were kept under regular LD followed by DD for 1 month. Ten days after release to DD, mice were treated with 5 doses of TAM (red triangles). (B) Locomotor activity was recorded using running wheels. The activity level was depicted as mean \pm SEM (shade, which indicates dark phase). 


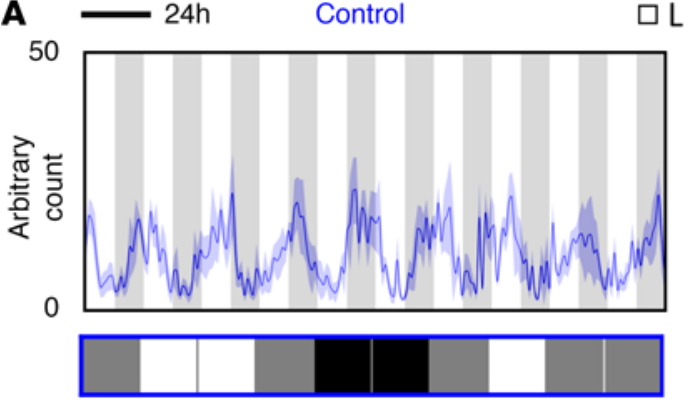

$\square$ Higher during light phase

B
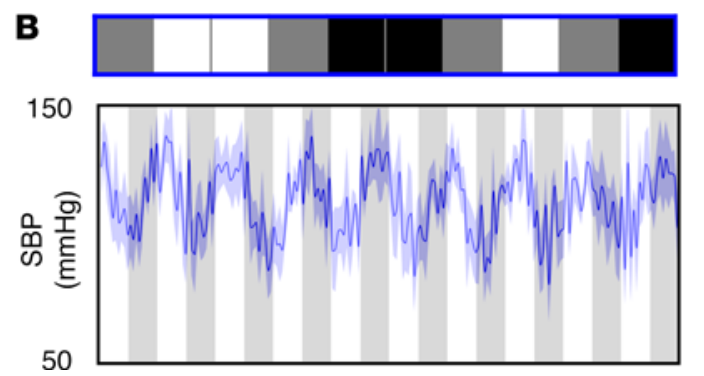

KO
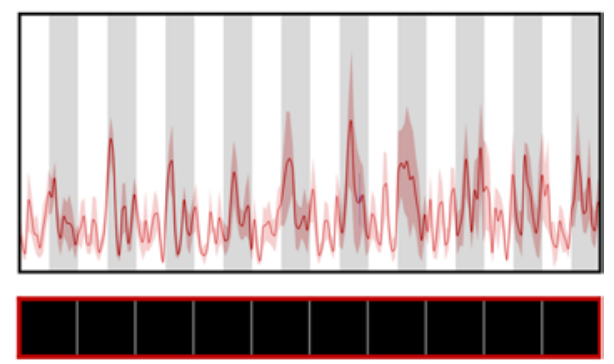

$\square$ No statistical difference
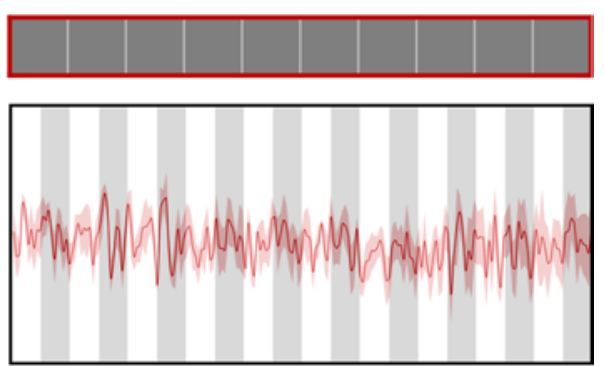

Figure 3. Locomotor activity and SBP under $L / D=10$ hours/10 hours condition.

(A) Locomotor activity and (B) SBP were measured using radiotelemetry. The average levels between light phase and dark phase within each cycle were compared. The differences were shown in the middle ( $n$ $=5$ for each group, Student's $t$ test): white square, significantly higher activity level or SBP during light phase $(P<$ $0.05)$; black square, significantly higher activity level or SBP during dark phase $(P<0.05)$; gray square, no statistical difference $(P>0.05)$ in activity level or SBP between light phase and dark phase.
Figure 4. Locomotor activity and SBP under $L / D=8$ hours $/ 8$ hours condition. (A) Locomotor activity and (B) SBP were measured using radiotelemetry. The average levels between light phase and dark phase within each cycle were compared. The differences were shown in the middle ( $n=5$ for each group, Student's $t$ test): white square, significantly higher activity level or SBP during light phase ( $P$ $<0.05)$; black square, significantly higher activity level or SBP during dark phase $(P<$ $0.05)$; gray square, no statistical difference $(P>0.05)$ in activity level or SBP between light phase and dark phase.
3 hours/ 3 hours schedule, the behavioral results showed that $\mathrm{KO}$ mice were more adaptive to these disruptions than were ctrl mice. This was reflected by calculation of the percentage of cycles that retained higher activity during the dark (active) phases (Figure 7).

Under these conditions, ctrl mice could not be entrained, as reflected by their continuous circadian rhythmicity in behavior irrespective of non-24-hour light/dark periods. For instance, under L/D = 10 hours/10 hours condition ( 8.3 days, 10 cycles), ctrl mice showed about 8 cycles instead of 10 cycles in behavior rhythmicity (Figure 3), while KO mice displayed higher activity levels in all dark phases. Similar results were also seen under $\mathrm{L} / \mathrm{D}=8 \mathrm{hours} / 8$ hours and randomized light/dark conditions. Interestingly, however, under L/D = 3 hours $/ 3$ hours condition, ctrl mice displayed higher activity levels mainly in dark phases, although there were still obvious circadian rhythms.

Dissociation between behavior and blood pressure in KO mice. Systolic BP (SBP) mirrored behavior in ctrl mice

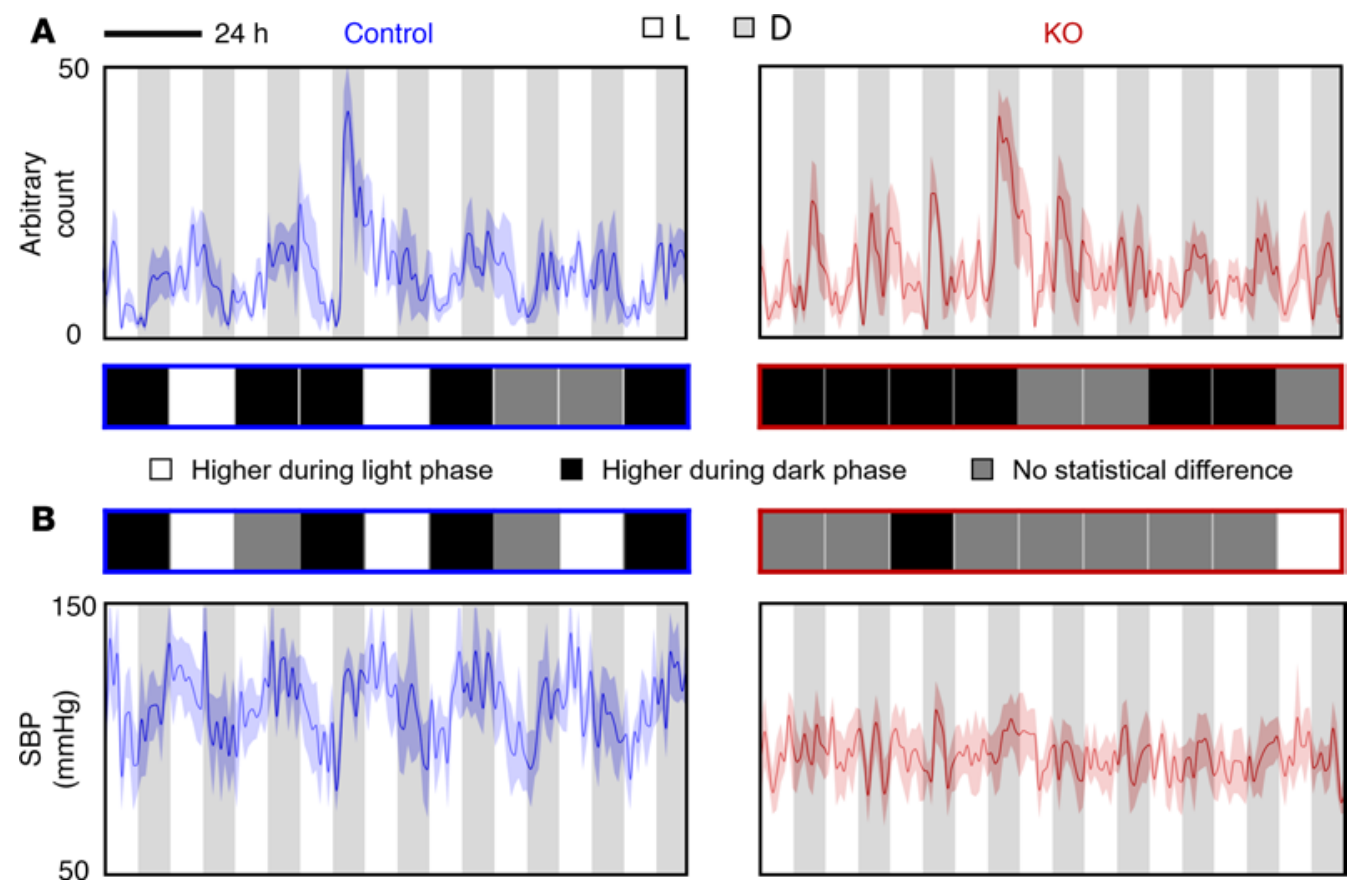


A

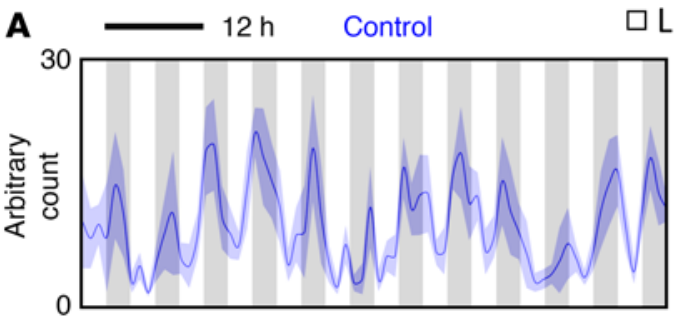

$\square \mathrm{D}$

KO
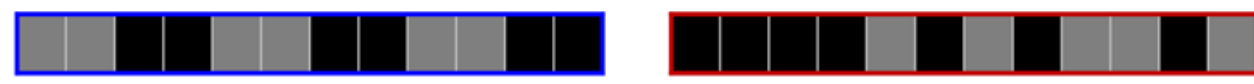

$\square$ Higher during light phase

Higher during dark phase

No statistical difference

B
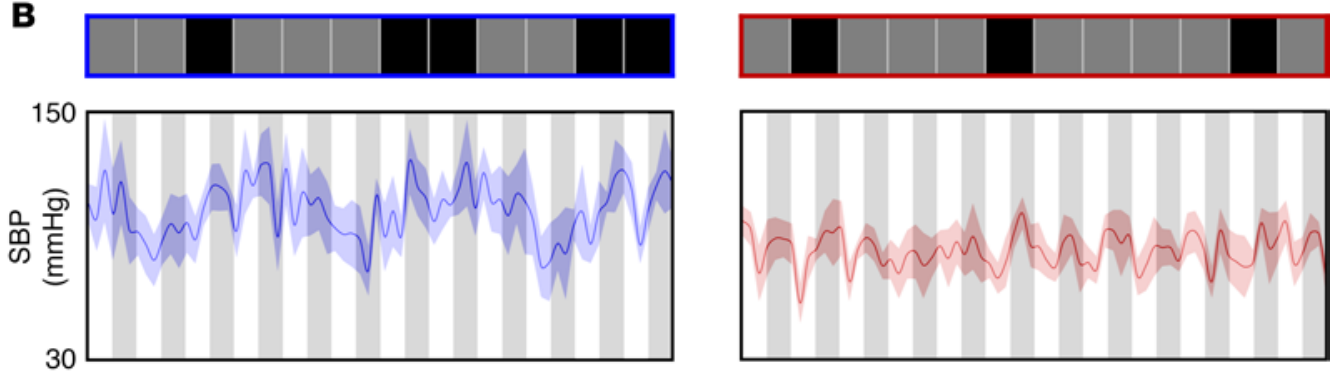

C

$24 \mathrm{~h} \quad \square \mathrm{L} \quad \square \mathrm{D}$
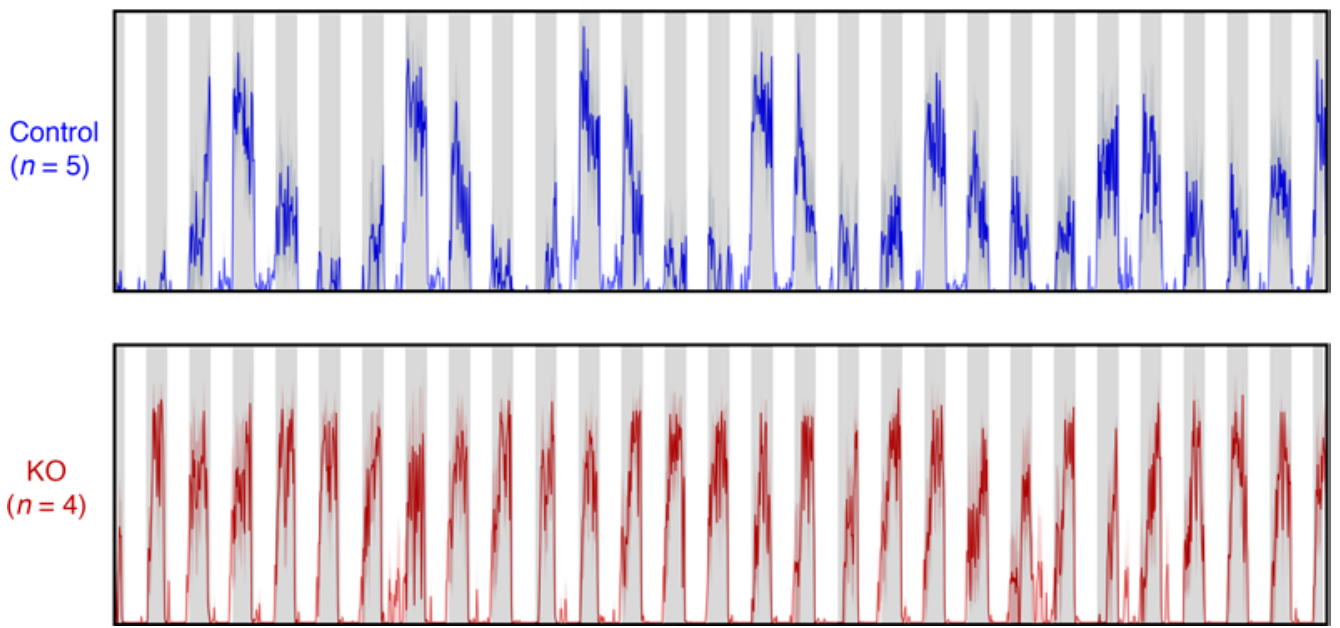

Figure 5. Locomotor activity and SBP under L/D = $\mathbf{3}$ hours/3 hours condition. (A) Locomotor activity and (B) SBP were measured using radiotelemetry. The average levels between light phase and dark phase within each cycle were compared. The differences were shown in the middle ( $n=5$ for each group, Student's $t$ test): white square, significantly higher activity level or SBP during light phase $(P<0.05)$; black square, significantly higher activity level or SBP during dark phase $(P<0.05)$; gray square, no statistical difference $(P>0.05)$ in activity level or SBP between light phase and dark phase. (C) Wheel running activity under $L / D=3$ hours $/ 3$ hours condition. The activity level was depicted as mean \pm SEM (shade, which indicates dark phase).

in that it failed to adapt to the disrupted schedules (Figures 3-6). Here, despite behavioral adaptation, SBP failed to retain rhythms in the KO mice exposed to the disrupted schedules (Figure 7).

Insulin tolerance test. Insulin sensitivity was markedly reduced by the abnormal schedules in ctrl mice as expected. However, concordant with behavioral adaptation, the $\mathrm{KO}$ mice were indistinguishable from unchallenged mice with respect to insulin sensitivity (Figure 8).

Body weight and fat weight. Body weight and epididymal fat weight were measured at the end of the experiments. No difference was found between ctrl and KO under either LD or DS conditions (Figure 9).

\section{Discussion}

Here we addressed the hypothesis that a functional molecular clock in adult mice would restrain their 
A
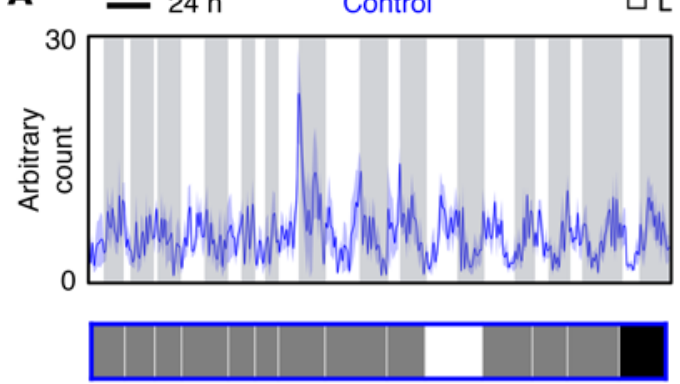

Higher during dark phase

\section{B}

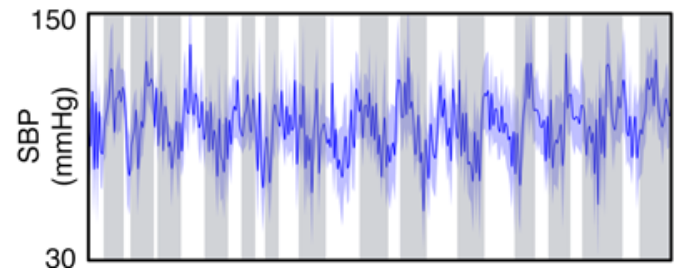

$\mathrm{KO}$
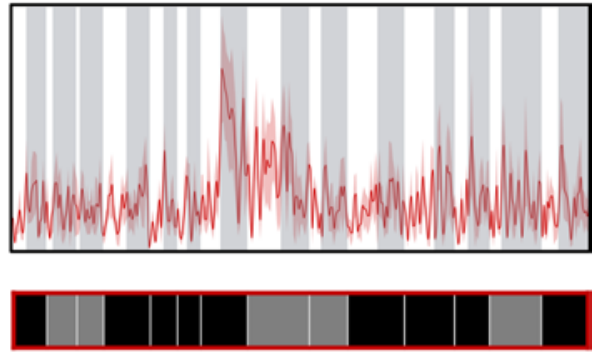

No statistical difference
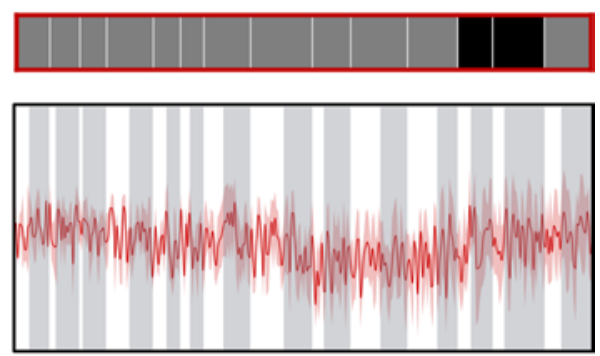

Figure 6. Locomotor activity and SBP under random light/dark conditions. (A) Locomotor activity and (B) SBP were measured using radiotelemetry. The average levels between light phase and dark phase within each cycle were compared. The differences were shown in the middle ( $n=5$ for each group, Student's $t$ test): white square, significantly higher activity level or SBP during light phase $(P<0.05)$; black square, significantly higher activity level or SBP during dark phase $(P<0.05)$; gray square, no statistical difference $(P>0.05)$ in activity level or SBP between light phase and dark phase.

ability to adapt to circadian disruption. We used mice in which the only nonredundant core clock gene, Bmal1, is deleted postnatally in a TAM-dependent manner. Previously we had shown that delaying such gene deletion until adulthood disrupted oscillatory gene expression, behavior, and BP as in conventional Bmal1-KO but that the 2 KOs diverged considerably in their impact on noncycling genes and various nonbehavioral phenotypes. These observations may reflect a role for Bmal1 independent of the core clock during development. Indeed, other core clock genes may also have off-target effects. For example, Period 2 (Per2), but not other clock genes, mediates a metabolic switch from fatty acid to glucose use in cardiomyocytes and thus protects the heart from ischemic injury in mice (10).

Here, the mice were subjected to a series of disrupted light schedules and a jet lag protocol. Generally, the KO mice adapted their behavior more readily to this circadian disruption than did the ctrls. Thus, ctrl mice could not be entrained under 10-hour/10-hour, 8-hour/8-hour, or randomized light/ dark regimes according to behavioral results, while the inducible-KO mice displayed higher activity levels in most dark phases of these schedules. The exception was the 3-hour/3-hour light/dark regime, in which the ctrl mice showed adaptation, displaying higher activity in the dark than the light phases.
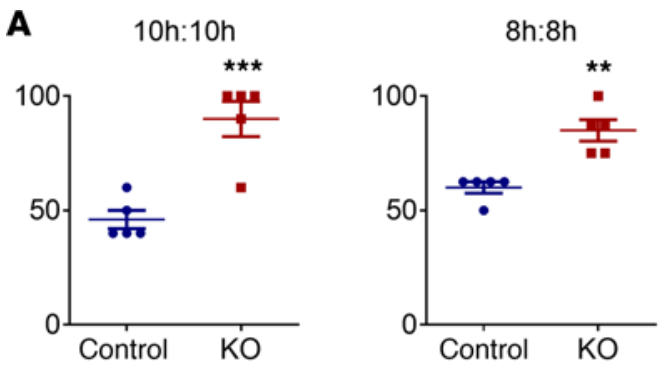

$3 h: 3 h$

Random

Figure 7. Percentage of cycles with higher activity level and SBP during dark phases. Percentage of cycles with higher (A) Locomotor activity ( $n=5$ for each group; ${ }^{* *} P<$ 0.01 ; ${ }^{* *} P<0.001$ ); (B) SBP ( $n=5$ for each group; ns, no significance).

B

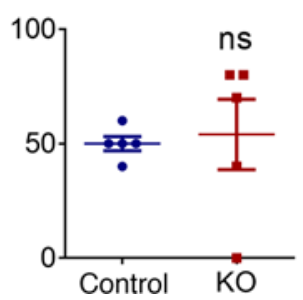

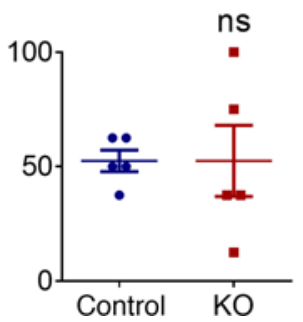
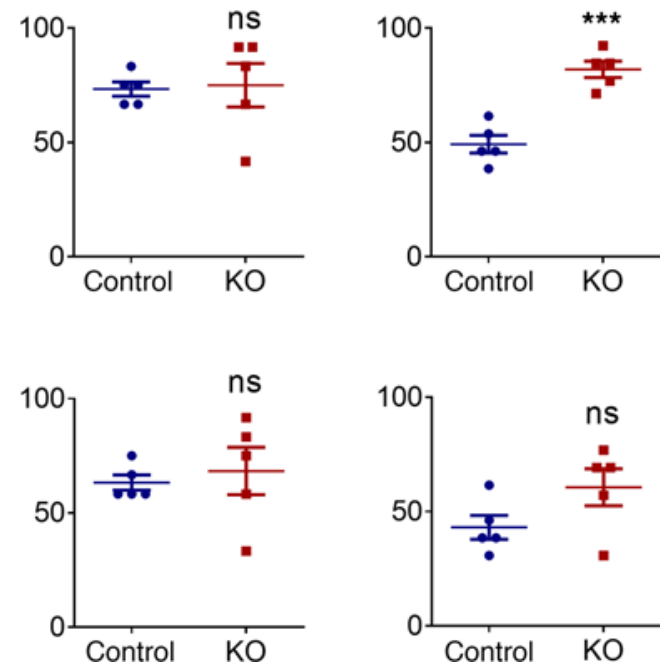

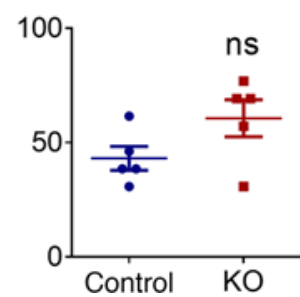



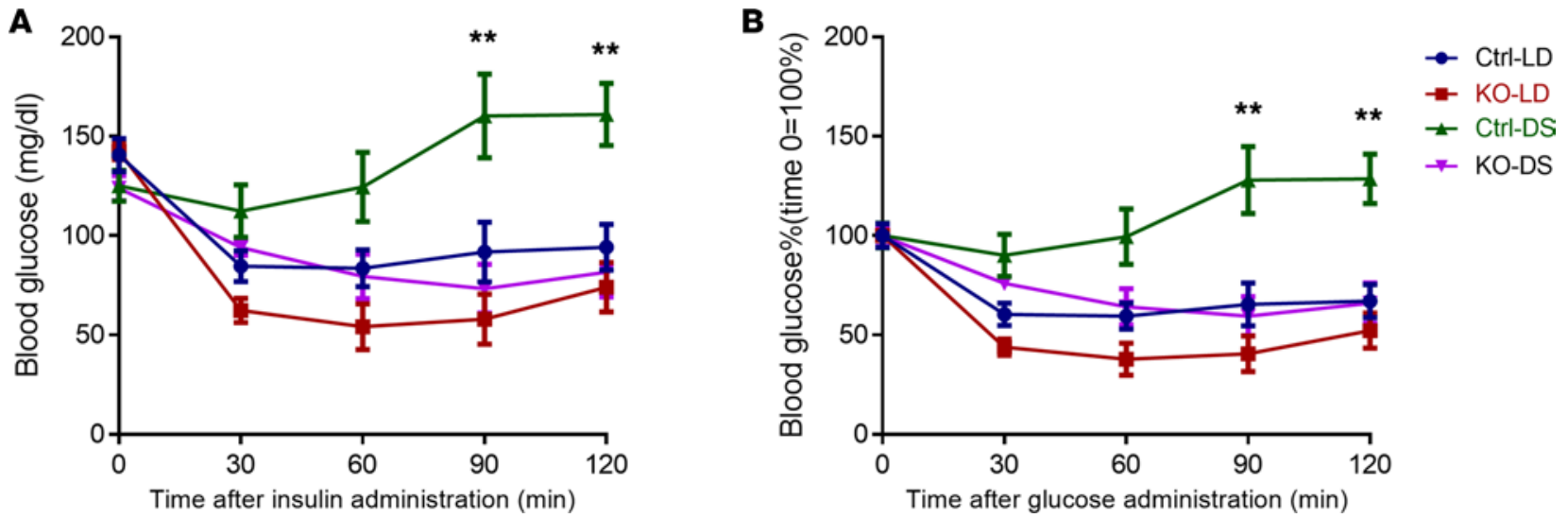

Figure 8. ITT in ctrl and KO mice under regular LD or DSs. (A) Blood glucose levels (mg/dl) after insulin injection. (B) ITT results were depicted as percentages. Basal blood glucose level of each group was defined as $100 \%\left(n=5\right.$ for each group; ${ }^{* *} P<0.01$, ctrl versus all other 3 groups, 2 -way ANOVA with Bonferroni's posttest).
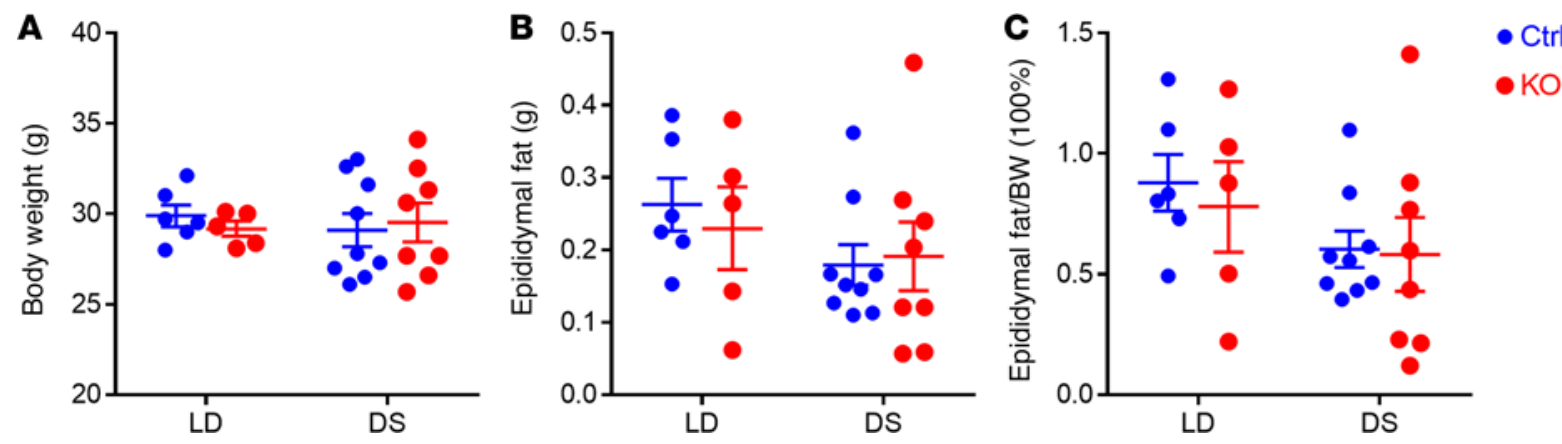

Figure 9. Body weight and epididymal fat weight of mice under regular LD or DSs. (A) Body weight, (B) epididymal fat weight, and (C) the ratio of epididymal fat weight to body weight. $n=5-9,2$-way ANOVA with Bonferroni's posttest. 


\section{Methods}

Mice. Bmalf $f^{f f}$ mice were obtained from Christopher A. Bradfield (University of Wisconsin, Madison, Wisconsin, USA) (18). The floxed mice were crossed with TAM-inducible (MilliporeSigma) universal Cre (EsrCre) mice (The Jackson Laboratory) to generate Bmalffff Esr-Cre mice. Both floxed and Cre mice were on a C57BL/ 6 background. To achieve global deletion of Bmall (KO), each mouse was treated with 5 mg TAM (in corn oil) per day by gavage for 5 consecutive days when they were 3 to 4 months old (9). Meanwhile, Cre mice were also treated with TAM as littermate ctrls. All mice in the study were male and single housed. They all had free access to food and water.

Telemetry recordings. The radiotelemetry device (TA11PA-C20, Data Sciences International) was implanted through catheterization of the carotid artery as previously described (12). Following a 1-week recovery, locomotor activity and BP were continuously recorded throughout the studies.

Study design. Radiotelemetry-implanted mice (5 for each genotype) were housed under the LD condition and then released into DD. For 10 days thereafter, mice were treated with 5 doses of TAM and followed while maintained under DD for 2 more weeks. Mice were then subjected to series of disrupted light/dark regimes, including $1, \mathrm{~L} / \mathrm{D}=10$ hours $/ 10$ hours for 200 hours $(10$ cycles $) ; 2, \mathrm{~L} / \mathrm{D}=8$ hours $/ 8$ hours for 144 hours ( 9 cycles); 3, L/D = 3 hours/3 hours for 72 hours (12 cycles); 4, LD for 72 hours (3 cycles); 5, randomized light/dark (random numbers were picked at https://www.random.org with lower limit at 2 and upper limit at 24) for 360 hours (14 cycles); 6, LD for 72 hours (3 cycles); 7, jet lag including two 8-hour delay shifts and one 8-hour advance shift at 3-day intervals (data were not collected because of the running out of the telemetry battery); 8, LD for 72 hours (3 cycles) (Figure 1).

Meanwhile, additional mice (10 ctrl, $8 \mathrm{KO}$ mice) without radiotelemetry implants were kept under normal LD condition or DSs as described above. At the end of the experiments, all mice were weighed and sacrificed for measurement of epididymal fat weight.

Running wheel. We used 2 independent sets of mice for activity recording using a running wheel system (Animal Activity Meter: OPTO-M4, Columbus Instruments). For set 1, mice were kept under DD for 5 days, then gavaged with TAM or corn oil for 5 consecutive days. For set 2 , mice were kept under regular LD for 7 days followed by L/D $=3$ hours/3 hours for 7 days.

Insulin tolerance test. An ITT was conducted after the series of DSs. Mice were fasted for 4 hours before the ITT. Blood from the tail vein was used for measurement of glucose level before (time 0 minutes) and at 30, 60, 90, and 120 minutes after intraperitoneal injection of insulin (1 U/kg) (Eli Lilly). Meanwhile, nonimplanted ctrl and KO mice kept under LD were included as controls for "normal" conditions.

Statistics. All statistical tests were 2 sided. Student's $t$ test was used when a single variable was compared between 2 groups. Two-way ANOVA with Bonferroni's posttest was used for weight and ITT measurements. The cutoff for significance was $P$ value less than 0.05 , denoted with an asterisk in figures. In all figures with error bars, the graphs depict mean \pm SEM

Study approval. Animal experiments performed in this study were approved by Dalian Medical University IACUC or by the University of Pennsylvania IACUC.

\section{Author contributions}

GY, LC, and GAF conceived and designed the research. GY, LC, JZ, and BR performed experiments. GY and LC analyzed the data. GY, LC, and GAF wrote the manuscript.

\section{Acknowledgments}

This work was supported by a grant from the NIH (U54TR001623), grants from the National Natural Science Foundation of China (81570643, 81670242, and 31871190), and a Merit Award from the American Heart Association. GAF is the McNeil Professor of Translational Medicine and Therapeutics.

Address correspondence to: Guangrui Yang, School of Bioengineering, Room 615, 2 Linggong Road, Ganjingzi District, Dalian, Liaoning, 116024, China. Phone: 86.411.84706806; Email: guangrui.yang@ hotmail.com. Or to: Garret A. FitzGerald, Institute for Translational Medicine and Therapeutics, Smilow Center for Translational Research, West Pavilion, 10th Floor, Room 116, 3400 Civic Center Blvd., Building 421, Philadelphia, Pennsylvania 19104-5158, USA. Phone: 215.898.1185; Email: garret@upenn.edu. 
1. Yang G, Paschos G, Curtis AM, Musiek ES, McLoughlin SC, FitzGerald GA. Knitting up the raveled sleave of care. Sci Transl Med. 2013;5(212):212rv3.

2. McDearmon EL, et al. Dissecting the functions of the mammalian clock protein BMAL1 by tissue-specific rescue in mice. Science. 2006;314(5803):1304-1308.

3. Bunger MK, et al. Progressive arthropathy in mice with a targeted disruption of the Mop3/Bmal-1 locus. Genesis. 2005;41(3):122-132.

4. Kondratov RV, Kondratova AA, Gorbacheva VY, Vykhovanets OV, Antoch MP. Early aging and age-related pathologies in mice deficient in BMAL1, the core component of the circadian clock. Genes Dev. 2006;20(14):1868-1873.

5. Marcheva B, et al. Disruption of the clock components CLOCK and BMAL1 leads to hypoinsulinaemia and diabetes. Nature. 2010;466(7306):627-631.

6. Zhang D, et al. The hepatic BMAL1/AKT/lipogenesis axis protects against alcoholic liver disease in mice via promoting PPAR a pathway. Hepatology. 2018;68(3):883-896.

7. Harfmann BD, Schroder EA, Kachman MT, Hodge BA, Zhang X, Esser KA. Muscle-specific loss of Bmal1 leads to disrupted tissue glucose metabolism and systemic glucose homeostasis. Skelet Muscle. 2016;6:12.

8. Paschos GK, et al. Obesity in mice with adipocyte-specific deletion of clock component Arntl. Nat Med. 2012;18(12):1768-1777.

9. Yang G, et al. Timing of expression of the core clock gene Bmall influences its effects on aging and survival. Sci Transl Med. 2016;8(324):324ra16.

10. Eckle T, et al. Adora2b-elicited Per2 stabilization promotes a HIF-dependent metabolic switch crucial for myocardial adaptation to ischemia. Nat Med. 2012;18(5):774-782.

11. Chen L, Yang G. Recent advances in circadian rhythms in cardiovascular system. Front Pharmacol. $2015 ; 6: 71$.

12. Yang G, Jia Z, Aoyagi T, McClain D, Mortensen RM, Yang T. Systemic PPAR $\gamma$ deletion impairs circadian rhythms of behavior and metabolism. PLoS One. 2012;7(8):e38117.

13. West AC, Smith L, Ray DW, Loudon ASI, Brown TM, Bechtold DA. Misalignment with the external light environment drives metabolic and cardiac dysfunction. Nat Commun. 2017;8(1):417.

14. van Oort BE, Tyler NJ, Gerkema MP, Folkow L, Blix AS, Stokkan KA. Circadian organization in reindeer. Nature. 2005;438(7071):1095-1096.

15. Stokkan KA, Mortensen A, Blix AS. Food intake, feeding rhythm, and body mass regulation in Svalbard rock ptarmigan. Am $J$ Physiol. 1986;251(2 Pt 2):R264-R267.

16. Revel FG, et al. The circadian clock stops ticking during deep hibernation in the European hamster. Proc Natl Acad Sci USA. 2007;104(34):13816-13820.

17. Kiessling S, Eichele G, Oster H. Adrenal glucocorticoids have a key role in circadian resynchronization in a mouse model of jet lag. J Clin Invest. 2010;120(7):2600-2609.

18. Johnson BP, et al. Hepatocyte circadian clock controls acetaminophen bioactivation through NADPH-cytochrome P450 oxidoreductase. Proc Natl Acad Sci USA. 2014;111(52):18757-18762. 